10,01

\title{
Возбуждение волн солитонного типа в кристаллах стехиометрии $A_{3} B$
}

\author{
(C) П.В. Захаров ${ }^{1}$, М.Д. Старостенков ${ }^{2}$, Е.А. Корзникова ${ }^{3}$, А.М. Ерёмин ${ }^{1}$, И.С. Луценко ${ }^{2}$, С.В. Дмитриев $^{3,4}$ \\ ${ }^{1}$ Алтайский государственный гуманитарно-педагогический университет им. В.М. Шукшина, \\ Бийск, Россия \\ ${ }^{2}$ Алтайский государственный технический университет им. И.И. Ползунова, \\ Барнаул, Россия \\ ${ }^{3}$ Институт проблем сверхпластичности металлов РАН, \\ Уфра, Россия \\ ${ }^{4}$ Национальный исследовательский Томский государственный университет, \\ Томск, Россия \\ E-mail: zakharovpv|@rambler.ru
}

Поступила в Редакцию 5 июня 2019 г.

В окончательной редакции 5 июня 2019 г.

Принята к публикации 18 июня 2019 г.

С использованием метода молекулярной динамики, рассмотрены кристаллы состава $\mathrm{A}_{3} \mathrm{~B}$, на примере $\mathrm{Ni}_{3} \mathrm{Al}$ и $\mathrm{Pt}_{3} \mathrm{Al}$, на предмет возможности возбуждения в них волн солитонного типа. Для описания межатомных взаимодействий использовались потенциалы, полученные методом погруженного атома. Показано, что при гармоническом внешнем воздействии возможно возбуждение волн солитонного типа в кристалле $\mathrm{Pt}_{3} \mathrm{Al}$, но не в $\mathrm{Ni}_{3} \mathrm{Al}$. Возникновение таких волн сжатия-растяжения обусловлено возбуждением вблизи зоны воздействия дискретных бризеров с мягким типом нелинейности, существование которых невозможно в кристалле $\mathrm{Ni}_{3} \mathrm{Al}$. Обнаруженные волны способны распространяться на тысячи нанометров вдоль кристалла $\mathrm{Pt}_{3} \mathrm{Al}$ без каких либо потерь формы и скорости движения. Форма полученной волны соответствует кинковому решению уравнения sin-Гордона. Совокупный объем энергии, переносимый волной, определяется количеством рядов атомов, вовлеченных в колебания, речь может идти о десятках и сотнях электрон-вольт.

Ключевые слова: дискретный бризер, солитон, уединенная волна, метод молекулярной динамики, нелинейность, колебания решетки.

DOI: 10.21883/FTT.2019.11.48426.507

\section{1. Введение}

Интерес к солитонам не угасает с момента их открытия и по сегодняшний день, поскольку с позиции солитонов удается трактовать явления, наблюдаемые в различных средах [1-5], а в области оптических солитонов развиваются технологии, реализуемые на практике $[6-11]$.

В отличии от уединенных волн на макро уровне на воде или в оптических и электрических системах, изучение уединенных волн на атомном уровне в твердых телах крайне затруднено в силу сложности их экспериментального наблюдения. В то же время имеются работы, в которых волны солитонного типа помогают трактовать различные эффекты. Так в работах $[12,13]$ предполагают, что именно солитонный механизм транспорта энергии от поверхности кристалла вглубь является одним из основных при ионном облучении кристаллов. В работах посвященных дискретным бризерам (ДБ) [14-20], которые также можно отнести к солитоноподобным объектам, рассматривается их способность осуществлять транспорт энергии. Говоря о ДБ, отметим, что их можно классифицировать по зависимости частоты от амплитуды колебаний. Так в случае если частота колебаний возрастает с увеличением амплитуды, то говорят о ДБ с жестким типом нелинейности, если же уменьшается - с мягким [21]. ДБ с жестким типом нелинейности чаще всего существуют на частотах выше оптической ветви фононного спектра (ФС) кристалла, хотя есть примеры, когда частота такого ДБ может отщепиться от акустической ветви ФС и существовать в щели спектра [22,23]. Такие бризеры локализованы на нескольких атомах и могут являться мобильными при наличии асимметрии в их профиле. ДБ с мягким типом нелинейности могут иметь частоты только в щели ФС кристаллов, они чаще всего локализованы на одном или на паре атомов, мобильностью при этом обычно не обладают. Среди экспериментальных работ, посвященных ДБ, можно выделить следующие. В работе [24] на основе исследования квазиодномерного кристаллического комплекса $\{[\mathrm{Pt}(e n) 2][\mathrm{Pt}($ en $) 2 \mathrm{Cl} 2](\mathrm{ClO} 4) 4\}$ (где (en) - этилендиамин) с помощью рамановского рассеяния делается вывод о возможности существования в нем некоторых локализованных динамических объектов, интерпретируемых как дискретные бризеры. При этом в работах [25-27] была предложена модель, результаты исследования которой хорошо согласуются с экспериментальными данными. Изучению мод, локализованных вдоль одного из пространственных измерений, в $\alpha$-фазе кристалла $U$ посвящены работы [28]. Авторы делают вывод о существовании в этом кристалле локализованных 

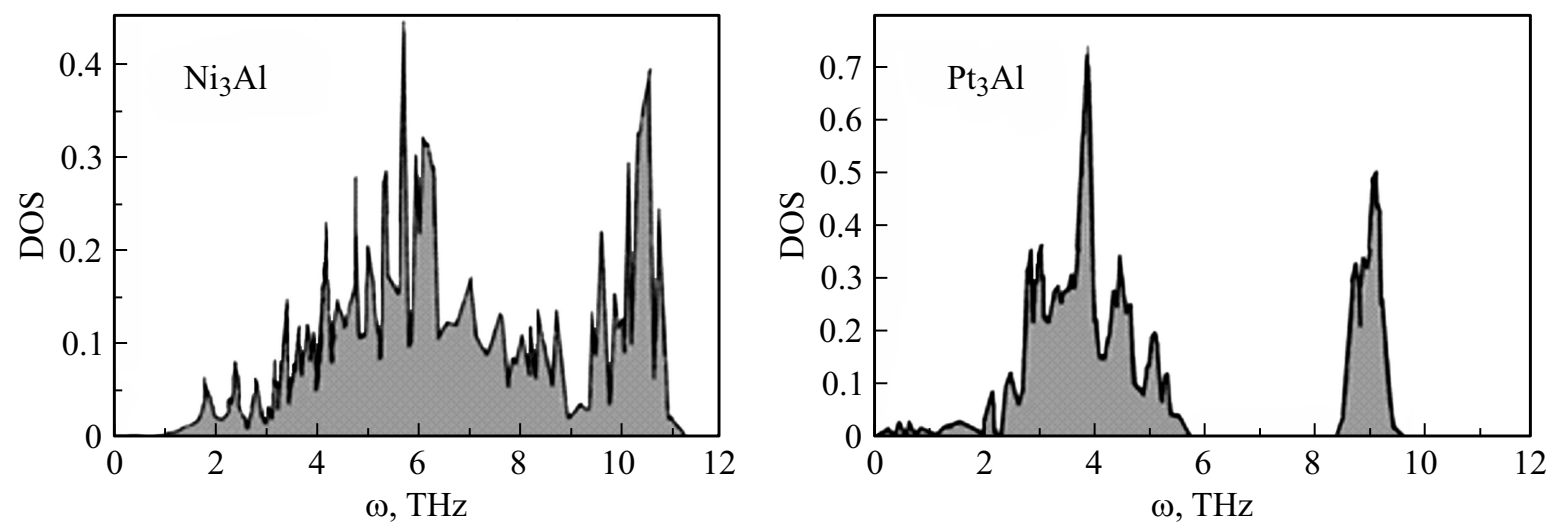

Рис. 1. Плотности фононных состояний кристаллов $\mathrm{Ni}_{3} \mathrm{Al}$ и $\mathrm{Pt}_{3} \mathrm{Al}$.

возбуждений, основываясь на результатах экспериментов по неупругому рентгеновскому рассеянию. В работах [28-30] обсуждаются экспериментальные данные, полученные с помощью неупругого рассеяния медленных нейтронов на колебаниях кристаллической решетки $\mathrm{NaI}$.

Приведенные работы, а также работы посвященные нелинейной супратрансмиссии [31-36], то есть передаче энергии кристаллу при периодическом воздействии на него на частотах вне фононного спектра кристалла, повышают интерес к таким механизмам транспорта энергии по кристаллу.

В данной работе методом компьютерного моделирования рассматривается возбуждение уединенных волн в кристаллах стехиометрического состава $\mathrm{A}_{3} \mathrm{~B}$ и устанавливается роль дискретных бризеров в данном процессе. Для сравнения выбраны кристаллы $\mathrm{Ni}_{3} \mathrm{Al}$ и $\mathrm{Pt}_{3} \mathrm{Al}$. Дело в том, что в кристалле $\mathrm{Pt}_{3} \mathrm{Al}$, в силу большой разницы атомных масс компонент, имеет место запрещенная зона в ФС, что обеспечивает возможность существования щелевых ДБ с мягким типом нелинейности [37,38], в то время как в $\mathrm{Ni}_{3} \mathrm{Al}$ такой щели нет, соответственно, отсутствуют условия для возбуждения таких ДБ.

\section{2. Модель и методика компьютерного эксперимента}

Рассматриваемая нами модель представляет собой объемный ГЦК кристалл стехиометрии $\mathrm{A}_{3} \mathrm{~B}$, содержащий от $3 \cdot 10^{5}$ до $2.5 \cdot 10^{6}$ частиц, взаимодействующих посредством потенциала, полученного методом погруженного атома (ЕАМ-потенциал) для $\mathrm{Pt}_{3} \mathrm{Al}$ [39] и $\mathrm{Ni}_{3} \mathrm{Al}$ [40]. Моделирование осуществлялось посредством пакета LAMMPS [41]. Полная энергия $E$ кристалла может быть выражена как $E=\frac{1}{2} \Sigma_{i, j, i \neq j} \varphi_{i j}\left(r_{i j}\right)+\Sigma_{i} F_{i}\left(\rho_{i}\right)$, где $\varphi_{i j}$ представляет парную энергию между атомами $i$ и $j$, отделенными друг от друга расстоянием $r_{i j}$, а $F_{i}-$ энергия вложения, связанная с вложенным атомом $i$ в локальном местоположении с электронной плотностью $\rho_{i}$. Электронную плотность можно рассчитать по формуле $\rho_{i}=\Sigma_{j, j \neq i} f_{j}\left(r_{i j}\right)$, где $f_{j}\left(r_{i j}\right)$ - электронная плотность на участке атома i, находящегося на расстоянии $r_{i j}$ от атома $j$.

Декартовы оси координат $X Y Z$ ориентированы вдоль кристаллографических направлений $\langle 100\rangle,\langle 010\rangle$ и $\langle 001\rangle$, соответственно. Размер ячейки варьировался в широком диапазоне с целью изучения механизмов возбуждения, распространения и взаимодействия уединенных волн. В модели вдоль осей $X, Y$ накладывались периодические граничные условия. Граничные условия на поверхностях нормальных оси $Z$ описаны ниже (вдоль этой оси рассматривалось движение солитонов). Отметим, что размер ячейки изменялся только вдоль оси $Z$, тем самым достигались линейные размеры ячейки вплоть до $2.4 \mu \mathrm{m}$. Вдоль осей $X$ и $Y$ размеры ячейки составляли $3.5 \mathrm{~nm}$ для $\mathrm{Ni}_{3} \mathrm{Al}$ и $4 \mathrm{~nm}$ для $\mathrm{Pt}_{3} \mathrm{Al}$.

Атомы расчетной ячейки были разбиты на три блока. Первый блок представлял собой 3-4 слоя атомов вблизи одной из поверхностей нормальных оси $Z$, которые осуществляли вынужденные колебания по гармоническому закону в направлении оси $Z$ с заданными частотой и амплитудой, взятыми из диапазонов указанных ниже. Во втором, основном блоке, изучалось поглощение кристаллом подведенной к нему энергии и ее распространение по кристаллу. На противоположном конце расчетной ячейки выделялся блок из 4-5 жестко зафиксированных слоев атомов. Это обеспечивало отсутствие движения всей модели кристалла.

Периодическое воздействие осуществлялось путем задания перемещений атомам первого блока по гармоническому закону $z(t)=A \sin (\omega t)$ вдоль оси $Z$ с частотами от 0.2 до $20 \mathrm{THz}$, и с амплитудами от 0.05 до $0.5 \AA$. Такой диапазон частот позволяет охватить весь спектр малоамплитудных колебаний атомов для рассматриваемых кристаллов. Время воздействия осуществлялось в интервале $1.5-8$ ps, тем самым обеспечивалось достаточное количество колебаний для разных частот внешнего воздействия.

Для рассматриваемых моделей кристаллов $\mathrm{Ni}_{3} \mathrm{Al}$ и $\mathrm{Pt}_{3} \mathrm{Al}$ были рассчитаны плотности фононных состояний (рис. 1). В расчетах использовался программный пакет 

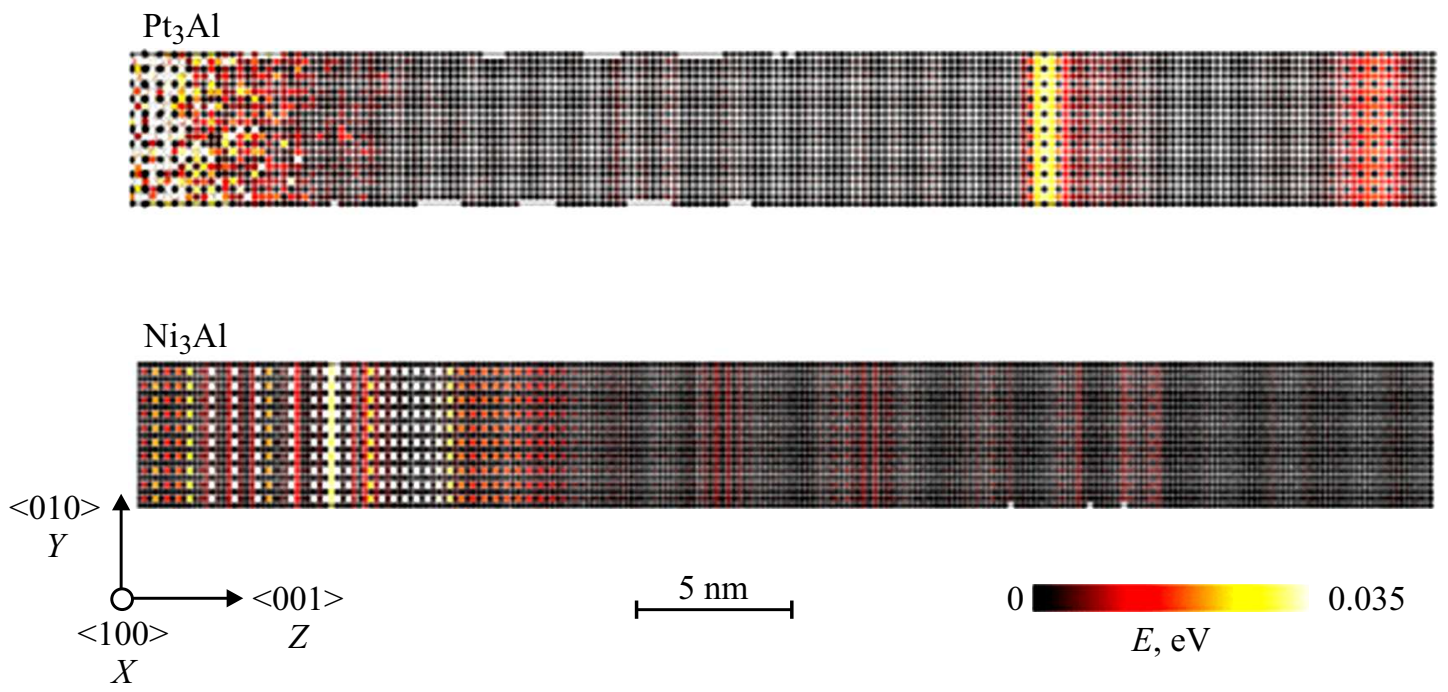

Рис. 2. Распределение кинетической энергии в кристаллах $\mathrm{Pt}_{3} \mathrm{Al}$ и $\mathrm{Ni}_{3} \mathrm{Al}$ через $8 \mathrm{ps}$ после начала эксперимента со следующими параметрами внешнего периодического воздействия $\omega=8 \mathrm{THz}, A=0.2 \AA$, время воздействия $t=2.5 \mathrm{ps}$.

LAMMPS, который включает необходимые для этих целей процедуры, базирующиеся на преобразовании Фурье автокорреляционных функций перемещений атомов от времени. Знание спектров кристаллов позволяет соизмерять частоты внешнего воздействия и собственные частоты их фононных колебаний, а также оценить вклад дискретных бризеров в процесс генерации уединенных волн.

\section{3. Результаты и обсуждения}

В результате проведенных расчетов было установлено, что в кристалле $\mathrm{Ni}_{3} \mathrm{Al}$ уединенные волны не возбуждаются при периодическом воздействии на конце расчетной ячейки с указанными частотами и амплитудами. Для примера приведем результаты эксперимента со следующими параметрами внешнего периодического воздействия $\omega=8 \mathrm{THz}, A=0.2 \AA$, время воздействия $t=2.5$ ps (рис. 2). Поведение кристалла $\mathrm{Pt}_{3} \mathrm{Al}$ отличалось от $\mathrm{Ni}_{3} \mathrm{Al}$, в нем формировались четко выраженные уединенные волны, бегущие вдоль кристалла (рис. 2). Характер этих волн зависел от амплитуды, частоты и продолжительности внешнего периодического воздействия. Так как в $\mathrm{Ni}_{3} \mathrm{Al}$ такой эффект не наблюдался, далее распространение волн будет изучаться только в кристалле $\mathrm{Pt}_{3} \mathrm{Al}$.

Рассмотрим механизм формирования полученных уединенных волн. В работах $[42,43]$ нами было показано, что периодическое воздействие на частотах близких к собственным частотам ДБ вызывает их возбуждение в непосредственной близости с областью воздействия, что выражается в резком увеличении амплитуды колебаний атомов Al. На рис. 3, $a$ приведены характерные графики зависимости $Z$-смещений атомов $\mathrm{Al}$ от положения равновесия в пограничном слое с областью периодиче- ского воздействия с амплитудой 0.1 и $0.2 \AA ̊$. Изменение координаты имеет колебательный характер, в момент достижения определенного значения начинает формироваться уединенная волна, дискретные бризеры отдают свою энергию, несмотря на продолжающееся внешнее воздействие, и далее процесс повторяется квазипериодически. На рис. $3, a$ для амплитуды воздействия $0.2 \AA$ на частоте $\omega=8 \mathrm{THz}$ сформировались две уединенные волны, которые отчетливо видны на рис. 2. Также данные результаты подтверждают наличие порогового значения амплитуды воздействия для возникновения эффекта супратрансмиссии. Так, при амплитуде $0.1 \AA$ и частоте $\omega=8 \mathrm{THz}$ ДБ не формировались, а энергия кристаллу практически не передавалась, так как частота воздействия лежала вне ФС кристалла. Минимальное значение амплитуды, при котором наблюдался ярко выраженный эффект формирования солитоноподобных волн, составило $0.15 \AA$.

Увеличение амплитуды внешнего воздействия приводит к быстрому разрушению приграничной области, однако, высокоамплитудные колебания атомов возникают в ближайшей упорядоченной части кристалла и таким же образом возбуждают уединенные волны.

Варьируя частоту воздействия (рис. $3, b$ ) при амплитуде $0.2 \AA$, установлено, что данный эффект возникает только на частотах вне $Ф$ кристалла, в том числе и для частот выше оптической ветви спектра, а именно для частот кратных частотам дискретных бризеров $(7-8 \mathrm{THz}[38])$ в рассматриваемом кристалле. При этом чем больше значение частоты воздействия, тем большее количество энергии может быть сосредоточено на уединенной волне.

Более подробно рассмотрим характер распределения кинетической энергии для одиночной волны возбужденной при следующих параметрах внешнего воздействия: $\omega=7 \mathrm{THz}, \quad A=0.2 \AA$, время воздействия $t=1.5 \mathrm{ps}$. 


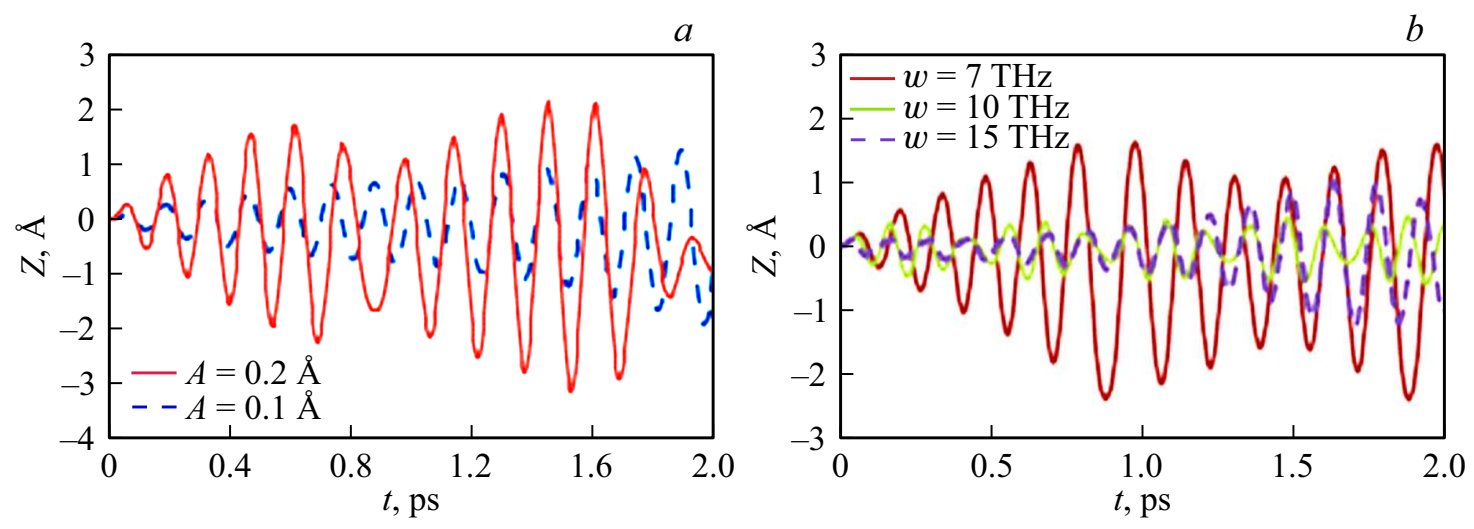

Pис. 3. Z-компонента атомных смещений атомов $\mathrm{Al}$, расположенных вблизи области внешнего воздействия как функция времени: для амплитуд воздействия 0.1 и $0.2 \AA$ на частоте $\omega=8 \mathrm{THz}$; для амплитуды воздействия $0.2 \AA$ на частотах $\omega=7,10$ и $15 \mathrm{THz}$. Результаты для $\mathrm{Pt}_{3} \mathrm{Al}$.
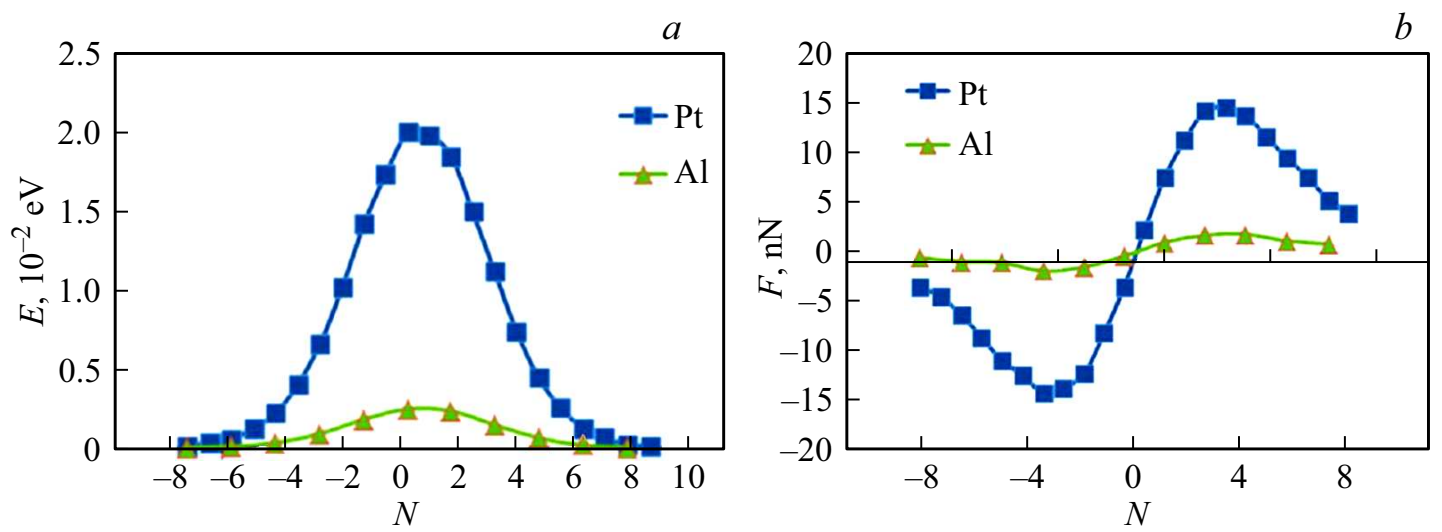

Рис. 4. Распределение энергии $(a)$ и $Z$-компоненты силы $(b)$ в уединенной волне для компонент сплава вдоль оси $Z$. Параметры внешнего воздействия: $\omega=7 \mathrm{THz}, A=0.2 \AA$, время воздействия $t=1.5 \mathrm{ps}$. Результаты для $\mathrm{Pt}_{3} \mathrm{Al}$.

В данном случае формировалась только одна волна, имеющая колоколообразный профиль распределения энергии (рис. 4). Отметим, что основная доля кинетичекой энергии сосредоточена на атомах $\mathrm{Pt}$, в то время как на атомы Al приходится незначительная ее часть. На рис. 4, $a$ приведен энергетический профиль волны, что позволяет оценить величину запасенной энергии. На рис. 4, $b$ дан график сил, действующих на атомы вдоль направления распространения волны, то есть вдоль оси Z. Видно, что в передней части волны материал испытывает сжатие, за которой идет область такого же по величине растяжения. После прохождения такой волны материал оказывается в естественном ненагруженном состоянии. Переноса массы такая волна не осуществляет, в отличие от хорошо известных волн сжатия.

Как уже отмечалось, амплитуда воздействия оказывает существенное влияние на характер формируемых волн и их свойства. Для наглядности рассмотрим профиль волн в зависимости от амплитуды воздействия для рядов атомов Pt.

На рис. 5, $a-c$ приведено распределение энергии атомов Pt вдоль кристаллографического направления $\langle 001\rangle$, вдоль которого распространяются рассматривае- мые волны. Для амплитуд менее $0.25 \AA$ характерно формирование уединенной волны состоящей из одного пика. С увеличением амплитуды воздействия до величин $0.25-0.325 \AA$ наблюдается формирование двухтрех пиков, распространяющихся вместе. Дальнейшее увеличение амплитуды приводит к устойчивому формирование двух пиков (рис. 5,c). Рассматривая причины такого поведения, вычислим кинетическую энергию, сосредоточенную вдоль одного ряда атомов Pt, такая зависимость от амплитуды воздействия приведена на рис. 5, $d$. Характерными являются две ступеньки при амплитудах 0.25 и $0.4 \AA$. Такое поведение вызвано изменением времени разрушения атомных слоев граничащих с областью воздействия (рис. 6). Для амплитуд менее $0.25 \AA$ разрушение кристаллической структуры не происходит, на атомах алюминия происходит возбуждение дискретных бризеров, после чего они передают часть энергии кристаллу в виде уединенной волны без нарушения структуры. Увеличение амплитуды приводит к разрушению кристаллической структуры атомных слоев, так при амплитуде $0.25 \AA$ данный процесс начинается через 0.7 ps после начала воздействия, а для амплитуд более $0.4 \AA$ через 0.4 ps. Формируется область с неупоря- 

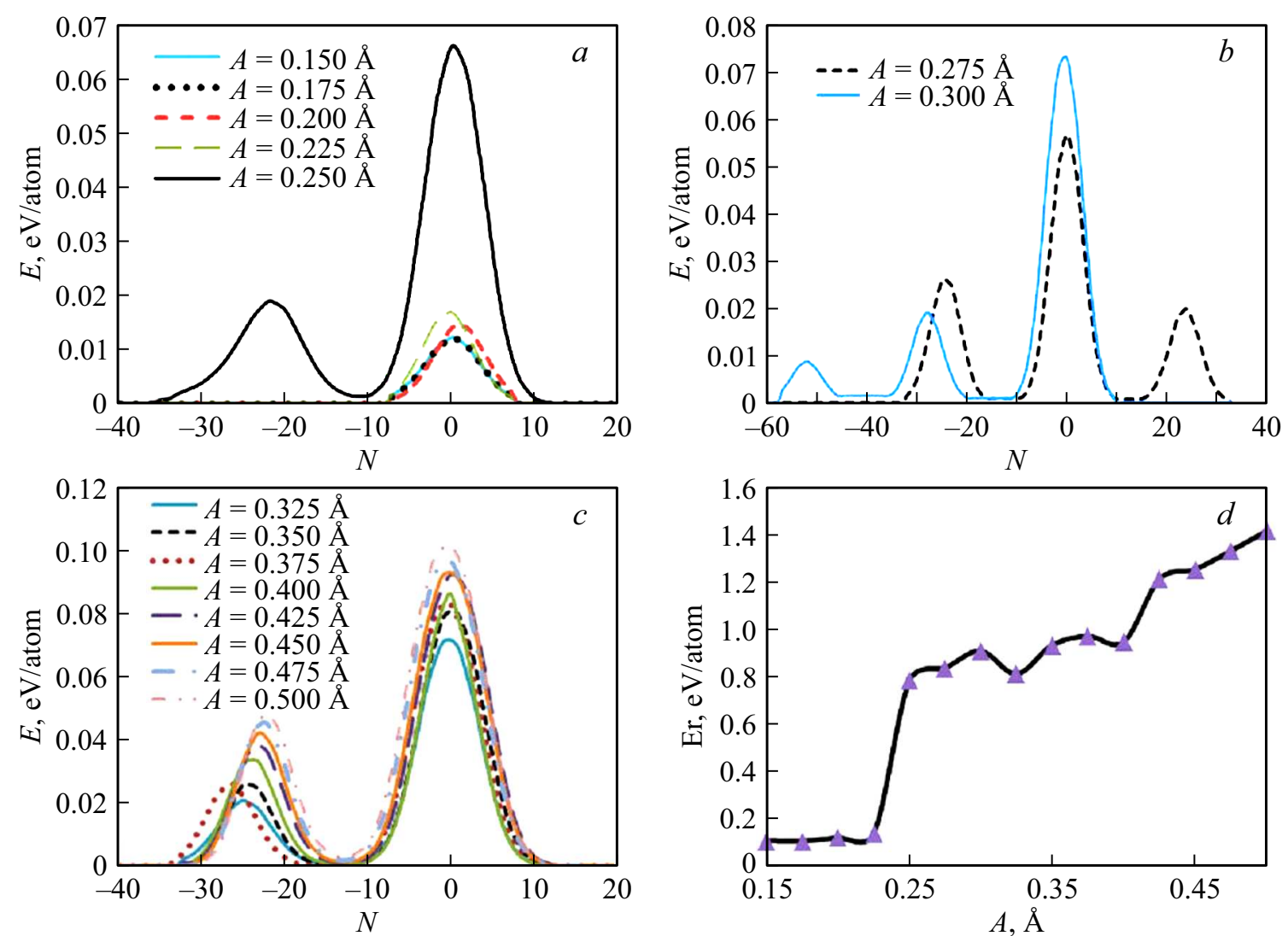

Рис. 5. $a-c$ Профиль кинетической энергии атомов ряда Pt вдоль бегущей уединенной волны в зависимости от амплитуды внешнего воздействия; $d$ - зависимость суммарной энергии атомов ряда Рt вдоль волны от амплитуды внешнего воздействия.

доченной структурой (расплав кристалла) между зоной воздействия и основным кристаллом. Образуется своеобразная подушка, которая смягчает внешне воздействия и приводит к формированию дискретных бризеров уже на границе расплава и упорядоченного кристалла.

Оценка энергии уединенной волны для данной модели $(4 \times 4 \mathrm{~nm})$ составляет величины от $20 \mathrm{eV}$ до нескольких сотен $\mathrm{eV}$. Таким образом, такая волна, распространяясь по кристаллу, может оказывать существенное влияние на процессы, протекающие в кристаллах на атомном уровне.

В теории уединенных волн в твердых телах $[44,45]$ известно обобщенное уравнение синус-Гордона, имеющее вид $l_{0}^{2} p\left(1-\frac{V^{2}}{V_{k}^{2}}\right) u_{, q q}=\tilde{p}_{1} \sin u+\tilde{p}_{2} \sin u \cos u$, здесь $l_{0}-$ характеристическая длинна, $V-$ постоянная фазовая скорость, $V_{k}-$ фазовая скорость оптической моды. Решением данного уравнения при определенных граничных условиях являются уединенные и периодические волны. Известно решение вида: $U(q, t)=\frac{2 \alpha^{2} \cosh \varepsilon^{2}}{1+\alpha^{2} \cosh \varepsilon^{2}}$, где $\varepsilon=q-V t, \alpha-$ амплитудный множитель, $q-$ координата, $V-$ скорость движения солитона, $t-$ время. Подбирая соответствующие значения параметров данного решения, получен профиль уединенной волны, хорошо согласующийся с профилем солитона в кристалле $\mathrm{Pt}_{3} \mathrm{Al}$. Можно говорить, что полученная уединенная

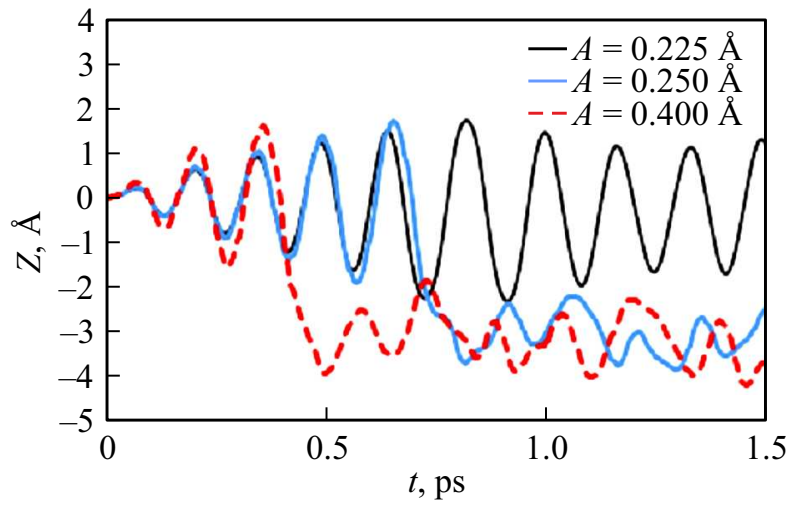

Рис. 6. Z-компонента смещений атомов $\mathrm{Al}$ от положений равновесия в непосредственной близости к области воздействия для амплитуд $0.225,0.25$ и $0.4 \AA$.

волна относится к классическим волнам солитонного типа. Ее профиль имеет колоколообразный вид, она нелинейна и распространяется с постоянной скоростью. Оценка величины скорости распространения такой волны дала значение порядка $4.4 \cdot 10^{3} \mathrm{~m} / \mathrm{s}$, независимо от параметров внешнего воздействия. Таким образом, сравнивая эту величину со скоростью звука в данной модели, которая составила $4.2 \cdot 10^{3} \mathrm{~m} / \mathrm{s}$, можно сказать, что 


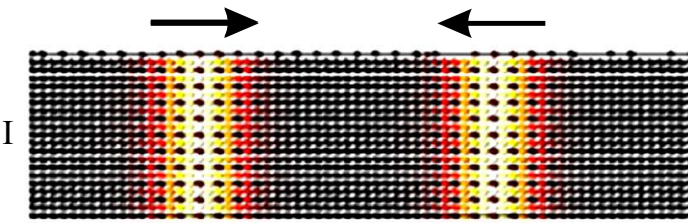

II
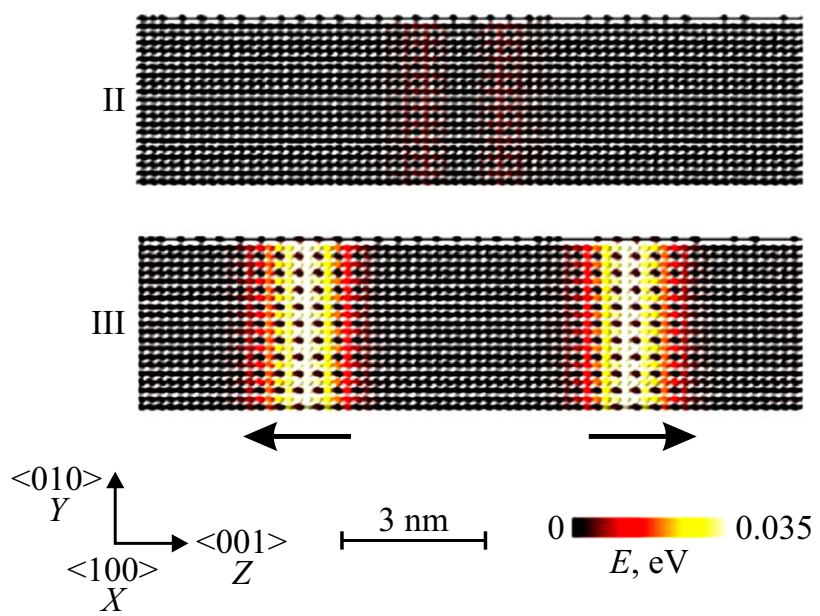

Pис. 7. Столкновение двух уединенных волн: I - до столкновения, II - момент столкновения, III - после столкновения.

полученная волна движется со сверхзвуковой скоростью в данной модели кристалла $\mathrm{Pt}_{3} \mathrm{Al}$.

На рис. 7 приведено распределение кинетической энергии вдоль кристалла при столкновении двух уединенных волн. Форма и скорость волн после столкновения остались неизменны.

Такие волны оказались также устойчивы к нагреву. Волна без существенного изменения своего профиля и скорости способна преодолевать нагретые области кристалла, по крайней мере, при температурах до $300 \mathrm{~K}$.

Установить максимально возможное расстояние, которое способен пройти солитон в рамках рассматриваемых моделей не удалось в виду значительных вычислительных временных затрат. Максимальное расстояние, которое было пройдено такой волной в моделях составило более $11 \mu \mathrm{m}$, при наложении жестких граничных условий на ячейку вдоль оси $Z$, после запуска уединенной волны. Солитон отражался от жестко зафиксированных атомов по краям ячейки и блуждал вдоль нее без каких либо признаков рассеяния энергии и изменения скорости движения.

\section{4. Заключение}

Таким образом, полученные уединенные волны способны распространяться на тысячи нанометров вглубь бездефектного кристалла без изменения формы и скорости. Совокупный объем энергии, переносимый волной, определяется количеством рядов атомов, вовлеченных в колебания, речь может идти о десятках и сотнях электрон-вольт. Данный механизм транспорта энергии по кристаллу, посредством уединенных волн, видится одним из наиболее эффективных, а механизм генерации таких волн относительно прост. Полученные результаты могут быть полезны при трактовке результатов натурных экспериментов при интенсивных внешних воздействиях на кристаллы, а также способны внести вклад в развитие представлений о роли дискретных бризеров в динамике решетки металлов и сплавов.

\section{Финансирование работы}

ЗПВ, СМД, ЕАМ, ЛИС благодарят за финансовую поддержку Российский фонд фундаментальных исследований и Администрацию Алтайского края в рамках научного проекта № 18-42-220002 p_a (получение исходных данных, написание статьи, подготовка иллюстраций). КЕА благодарит за финансовую поддержку грант Президента Российской федерации для государственной поддержки молодых российских ученых № МД-3639.2019.2 (обсуждение результатов, формулировка выводов).

\section{Конфликт интересов}

Авторы заявляют, что у них нет конфликта интересов.

\section{Список литературы}

[1] W.P. Su, J.R. Schrieffer, A.J. Heeger. Phys. Rev. Lett. 42, 1698 (1979).

[2] E. Luz, V. Lutsky, E. Granot, B.A. Malomed. Sci. Rep. 9, 4483 (2019).

[3] Z. Lan. Appl. Math. Lett. 94, 126 (2019).

[4] N. Tomita, A. Takahashi. Phys. Rev. B 99, 035203 (2019).

[5] M.G. Arabi, D.V. Sogut, A. Khosronejad, A.C. Yalciner. Coast. Eng. 147, 43 (2019).

[6] Y.S. Kivshar, G.P. Agrawal. Optical Solitons: From Fibers to Photonic Crystals Book Elsevier Inc., 540, ISBN: 978012410590-4 (2003).

[7] F. Lederer, G.I. Stegeman, D.N. Christodoulides, G. Assanto, M. Segev, Y. Silberberg. Phys. Rep. 463, 1 (2008).

[8] N.K. Efremidis, S. Sears, D.N. Christodoulides, J.W. Fleischer, M. Segev. Phys. Rev. E 66, 5 (2002).

[9] A.S. Raja, A.S. Voloshin, H. Guo. Nature Commun. 10, 680 (2019).

[10] O.V. Rudenko, C.M. Hedberg. Wave Motion 89, 104 (2019).

[11] A.N. Bugay, V.A. Khalyapin. Commun. Nonlinear Sci. Numer. Simul. 75, 270 (2019).

[12] С.Г. Псахье, К.П. Зольников, Р.И. Кадыров, Г.Е. Руденский, Ю.П. Шаркеев, В.М. Кузнецов. ПЖТФ 25, 7 (1999).

[13] A.A. Groza, P.G. Litovchenko, M.I. Starchik, V.I. Khivrych, G.G. Shmatko, V.I. Varnina. Nucl. Phys. At. Energy 11, 66 (2010).

[14] О.В. Бачурина, Р.Т. Мурзаев, А.С. Семенов, Е.А. Корзникова, С.В. Дмитриев. ФТТ 60, 978 (2018).

[15] А.С. Семенов, Ю.В. Бебихов, А.А. Кистанов. Письма о материалах 7, 77 (2017).

[16] А.А. Кистанов, А.С. Семенов, С.В. Дмитриев. ЖЭТФ 146, 869 (2014). 
[17] А.А. Кистанов, С.В. Дмитриев. ПЖТФ 39, 78 (2013).

[18] A. Shelkan, M. Klopov, V. Hizhnyakov. Phys. Lett. A 383, 1893 (2019).

[19] D. Saadatmand, D. Xiong, V.A. Kuzkin, A.M. Krivtsov, A.V. Savin, S.V. Dmitriev. Phys. Rev. E 97, 022217 (2018).

[20] D. Xiong, D. Saadatmand, S.V. Dmitriev. Phys. Rev. E 96, 042109 (2017).

[21] С.В. Дмитриев, Е.А. Корзникова, Ю.А. Баимова, М.Г. Веларде. УФН 186, 471 (2016).

[22] L.Z. Khadeeva, S.V. Dmitriev. Phys. Rev. B 81, 214306 (2010).

[23] J.A. Baimova, S.V. Dmitriev, K. Zhou. Europhys. Lett. 100, 36005 (2012).

[24] B.I. Swanson, J.A. Brozik, S.P. Love, G.F. Strouse, A.P. Shreve, A.R. Bishop, W.-Z. Wangl. Phys. Rev. Lett. 82, 3288 (1999).

[25] N.K. Voulgarakis, G. Kalosakas, A.R. Bishop, G.P. Tsironis. Phys. Rev. 64, 020301 (2001).

[26] G. Kalosakas, A.R. Bishop, A.P. Shreve. Phys. Rev. 66, 094303 (2002).

[27] D.K. Campbell, S. Flach, Yu.S. Kivshar. Phys. Today. 57, 43 (2004).

[28] M.E. Manley, A. Alatas, F. Trouwet. Phys. Rev. 77, 214305 (2008).

[29] M.E. Manley, M. Yethiraj, H. Sinn. Phys. Rev. Lett. 96, 125501 (2006).

[30] M.E. Manley, A.J. Sievers, J.W. Lynn. Phys. Rev. 79, 134304 (2009).

[31] F. Geniet, J. Leon. Phys. Rev. Lett. 89, 134102 (2002).

[32] R. Khomeriki, S. Lepri, S. Ruffo. Phys Rev. E 70, 066626 (2004).

[33] I. Evazzade, I.P. Lobzenko, E.A. Korznikova, I.A. Ovid'ko. Phys. Rev. B 95, 035423 (2017).

[34] B. Yousefzadeh, A.S. Phani. JSV 380, 242 (2016).

[35] J. Leon. Phys. Lett. A 319, 130 (2003).

[36] L. Ponson, N. Boechler, Y.M. Lai, M.A. Porter, P. Kevrekidis, C. Daraio. Phys. Rev. E 82, 021301 (2010).

[37] П.В. Захаров, М.Д. Старостенков, С.В. Дмитриев, Н.Н. Медведев, А.М. Ерёмин. ЖЭТФ 148, 252 (2015).

[38] P.V. Zakharov, E.A. Korznikova, S.V. Dmitirev, E.G. Ekomasov, K. Zhou. Surf. Sci. 679, 1 (2019).

[39] X.W. Zhou, R.A. Johnson, H.N.G. Wadley. Phys. Rev. B 69, 144113 (2004).

[40] G.P. Purja Pun, Y. Mishin. Phil. Mag. 89, 3245 (2009).

[41] Information on LAMMPS Molecular Dynamics Simulator on http://lammps.sandia.gov

[42] Н.Н. Медведев, М.Д. Старостенков, П.В. Захаров, О.В. Пожидаева. ПЖТФ 37, 7 (2011).

[43] Н.Н. Медведев, М.Д. Старостенков, П.В. Захаров, А.В. Маркидонов. Письма о материалах 3, 34 (2013).

[44] Э.Л. Аэро, А.Н. Булыгин. Вычислительная механика сплошных сред 1, 14 (2008).

[45] Э.Л. Аэро, А.Н. Будыгин. Вычислительная механика сплошных сред 2, 19 (2009).

Редактор К.В. Емиев 